\title{
Latitudinal and temporal variation of equatorial ionospheric irregularities determined from GPS scintillation observations
}

\author{
M. A. Cervera ${ }^{1}$ and R. M. Thomas ${ }^{2}$ \\ ${ }^{1}$ Intelligence, Surveillance and Reconnaissance Division, Defence Science and Technology Organisation, PO Box 1500, \\ Edinburgh, S.A. 5111, Australia \\ ${ }^{2}$ Box 154 , Cockatoo Valley, S.A. 5351, Australia
}

Received: 10 April 2006 - Revised: 19 October 2006 - Accepted: 27 November 2006 - Published: 21 December 2006

\begin{abstract}
In this paper we investigate the spatial and temporal distribution of ionospheric irregularities in the South-East Asian longitude sector by analysing the scintillation of GPS signals received at ground based stations. The data used for this work were collected during 1998 to 2002 with our regional network of GPS based scintillation monitors (Thomas et al., 2001).

The aim of this analysis is to better understand the morphology and climatology of ionospheric irregularities in our region. Interesting differences between the north and south anomalies are noted when we examine the latitudinaltemporal variation of irregularity activity. We investigate the possible causes of these differences and note that variations in the irregularity seeding mechanism and in the background ionosphere at the two anomalies could be important.

Phase screen diffraction theory is used to analyse our scintillation data and we note problems with this theory for raypaths with large off-zenith angles. The height of the phase screen is another important issue. We discuss the implications for models which rely on phase screen diffraction theory.
\end{abstract}

Keywords. Ionosphere (Equatorial ionosphere; Ionosperic irregularities; General or miscellaneous)

\section{Introduction}

Radio-wave signals which traverse the Earth's ionosphere are subject to various effects. Under quiescent conditions (i.e. the ionosphere is not turbulent) such signals experience the well know group delay and carrier advance due to the non-unitary refractive index of the medium. As the ionosphere is inhomogeneous, refraction of the radio-wave signal occurs. When the ionosphere becomes turbulent through the generation and evolution of irregularities, more severe effects are imposed upon the signals. Diffraction of the signals oc-

Correspondence to: M. A. Cervera

(manuel.cervera@dsto.defence.gov.au) curs and as the irregular medium drifts with respect to the ray path the diffraction pattern observed at the receiver fluctuates temporally. At HF this is manifest as spread-F in radar observations. For trans-ionospheric signals such as those from GPS satellites it is manifest as amplitude and phase scintillation imposed upon the signals. The two main regions of the ionosphere where irregularities occur are the polar and equatorial zones. The physical processes involved are very different and we focus solely on the latter.

The equatorial ionosphere is that region bounded by $\sim \pm 20^{\circ}$ magnetic latitude with the Appleton anomaly peaking at $\sim \pm 15^{\circ}$. Irregularities may form in this region of the ionosphere $1-2 \mathrm{~h}$ post sunset, and the irregularity generating region moves westward with the sunset terminator. The irregularities, in the form of bubbles of depleted ionization, are generated at or near the magnetic equator in the bottom-side of the F2 layer, rise and map poleward along the magnetic field lines until they reach the Appleton anomaly regions north and south of the equator. Heights of up to $1000 \mathrm{~km}$ may be attained. The bubbles are large structures and as they map down the field lines they break down into a spectrum of smaller irregularities. Once formed the irregularities subsequently drift eastward with the neutral wind.

The physical process which generates the bubbles is a Rayleigh-Taylor like instability (Kelley, 1989). Chemical processes which control recombination in the post sunset ionosphere produce a sharp gradient in the vertical electron density profile. Perturbations (e.g. due to acoustic gravity waves or the equatorial post-sunset vortex) in this region become unstable at the magnetic equator due to east-west polarisation electric fields which form within the perturbation as a consequence of the zonal current flow. The resultant $\delta \boldsymbol{E} \times \boldsymbol{B}$ forces at the magnetic equator cause the perturbations to become unstable.

Gravity waves in the neutral atmosphere have long been recognised as the generating mechanism of travelling ionospheric disturbances (TIDs) and the case for their role as the seeding mechanism of ionospheric irregularities is steadily strengthening. Whitehead (1971) first proposed that gravity

Published by Copernicus GmbH on behalf of the European Geosciences Union. 
waves can produce perturbations in the ionosphere under the conditions of spatial resonance. Here the requirement is that the phase speed of the gravity wave is equal to the drift speed of the ionization. Huang and Kelley (1996a,b) extensively modelled the physical processes whereby gravity waves seeded the initial perturbations in the bottomside night time ionosphere and their subsequent evolution through the Rayleigh-Taylor instability amplification to produce plasma bubbles and plumes. They not only demonstrated the importance of the gravity wave seeding mechanism of plasma bubbles but also that spatial resonance was not required for their generation (although spatial resonance could speed up the process). Extensive observations of equatorial plasma bubbles by radar (e.g. Kelley et al., 1981; Hysell et al., 1990; Kelley and Hysell, 1991; Huang et al., 1993), satellite borne ion drift sensors (e.g. Singh et al., 1997), and optical observations of $\mathrm{F}$ region night-glow emissions (e.g. Fagundes et al., 1999; Sinha and Raizada, 2000) have increasingly strengthened the hypothesis that gravity waves are a seed mechanism. Recently Nicolls and Kelley (2005) observed wave activity several hours prior to the onset of the conditions suitable for plasma instabilities. Once these conditions were met the perturbations rapidly generated ionospheric irregularities. This new observation has added additional compelling evidence for gravity wave seeding of equatorial plasma bubbles.

A further controlling factor of the generation of equatorial ionospheric irregularities is the orientation of the solar terminator with respect to the geomagnetic field lines (Tsunoda, 1985). When the terminator is aligned with the geomagnetic field, the longitudinal gradient in the E-region Pedersen conductivity maximises. This causes an enhancement in the eastward electric field and, in turn, the polarisation electric fields within any ionospheric perturbations. Consequently the strength of the irregularities maximises during these times. In the south-east Asian longitude sector the magnetic declination is small and so the maxima in the irregularity strength occur at the equinoxes.

The extent of the source region and the altitude and latitudinal distribution of irregularities within the source region are poorly understood as is the mechanism which governs the mapping of the bubbles down the magnetic field lines. These issues formed one of the motivating factors behind the establishment of our network of scintillation monitors in the South-East Asian region. Other motivating factors include the evaluation of the robustness of GPS integrity under scintillation conditions (Thomas et al., 2004), and the validation of scintillation models (Cervera et al., 2001).

\section{GPS scintillation monitor network}

Scintillation data are provided by Ionospheric Scintillation Monitors (ISM). The ISMs are based on Novatel 951 GPS single frequency (L1) receivers which have been modified to process raw data sampled at $50 \mathrm{~Hz}$ and calculate various parameters which characterise the observed scintillation. The ISMs are stationed at 6 separate locations in the SouthEast Asian region, a detailed description of the the network is given by Thomas et al. (2001). Briefly, Fig. 1 displays the locations of the GPS based ISMs within the network together with their coverage circles. Darwin, Chiang-Rai, Marak Parak, and Pare Pare also have dual frequency Novatel Millenium GPS receivers for the purpose of measuring TEC. However, these will not be described here.

In this paper we will present data from three stations only viz. Chiang-Rai, Pare Pare and Marak Parak. These stations are respectively located under the north and south anomaly crests, and the magnetic equator.

The ISMs $\log$ data automatically at 1 minute intervals throughout the day. Quantities which are logged include the amplitude and phase scintillation indices $S_{4}$ and $\sigma_{\phi}$, and phase spectral parameters $T$ (strength at $1 \mathrm{~Hz}$ ) and $p$ (index of power-law decay). Raw $50 \mathrm{~Hz}$ data may also be recorded non-routinely if desired. Detailed descriptions of the ISMs may be found in Van Dierendonck et al. (1993) and Van Dierendonck et al. (1996).

The $S_{4}$ index is given by the standard deviation of the signal power normalised by its mean and typically lies in the range 0 to 1 , whereas $\sigma_{\phi}$ is the standard deviation of the phase which has been detrended to remove total electron content (TEC) variations. Rino (1979) has shown that the phase variance may also be given by

$\sigma_{\phi}^{2}=\frac{2 T}{(p-1) f_{c}^{p-1}}$,

where $f_{c}$ is the cutoff frequency of the detrend filter.

The phase data from these ISMs can, on occasions, be subject to external noise severe enough to render them unusable. Care must be taken to ensure that these data are discarded. Of much greater concern was the unreliability of the value of $p$ measured by the ISMs. As we shall see in the next section, $p$ is crucial for the calculation of the strength of the irregularities. The problem is due to the ISM firmware which performs a linear least squares fit to the phase spectrum in order to calculate $p$. Unfortunately, this fit often extends down into the system noise which biases $p$ towards lower values and the problem becomes progressively worse as the level of phase scintillation decreases.

Given the importance of $p$, we need an alternative method for producing reliable values. Equation (1) may be used to this end when the ISMs are not subject to phase noise. Rearranging this equation yields

$$
\frac{2 T \log \left(f_{c}\right)}{\sigma_{\phi}^{2}}=(p-1) \log \left(f_{c}\right) e^{(p-1) \log \left(f_{c}\right)},
$$

whence

$p=W\left(\frac{2 T \log \left(f_{c}\right)}{\sigma_{\phi}^{2}}\right) / \log \left(f_{c}\right)+1$, 


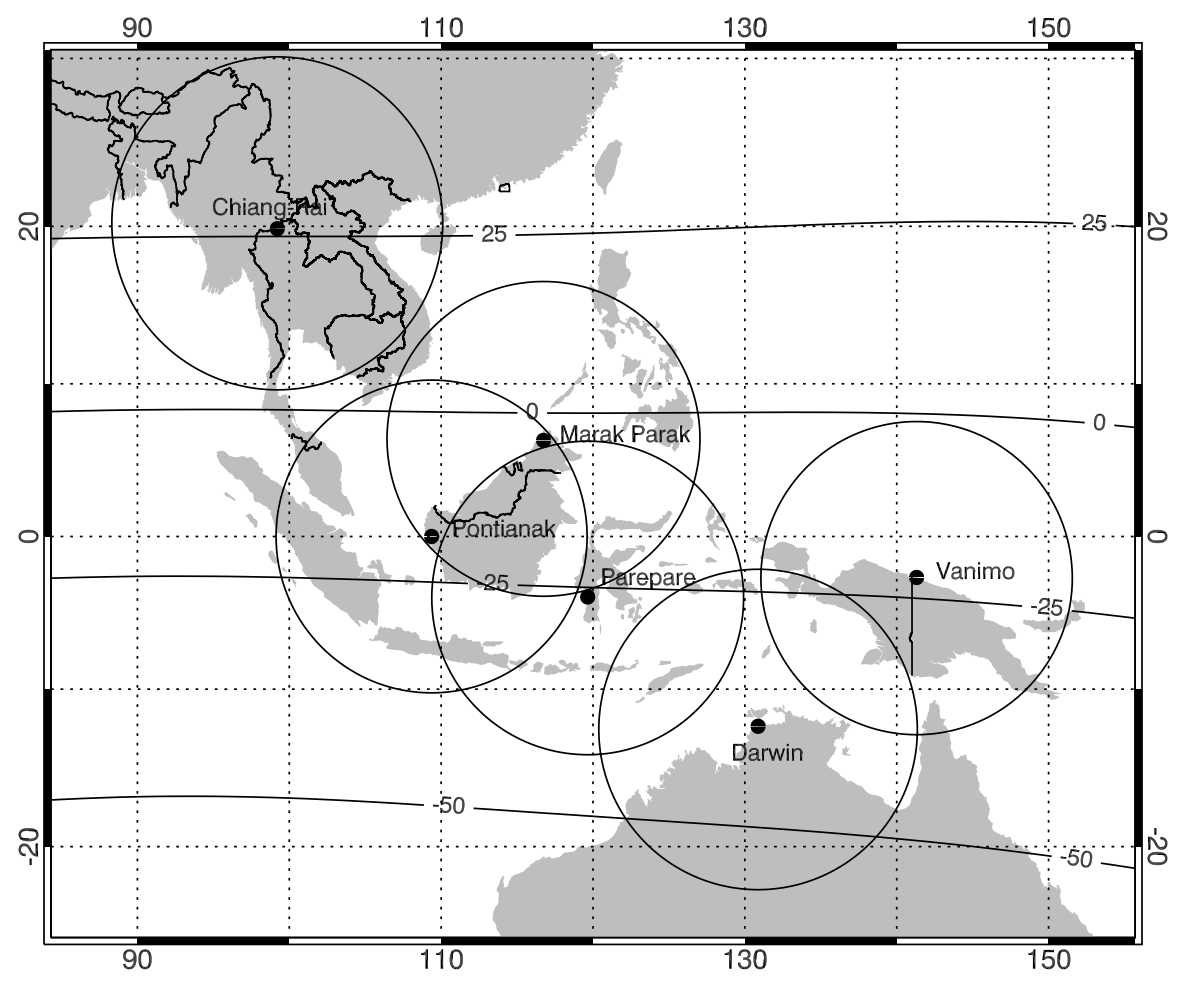

Fig. 1. Locations of GPS receiver network stations. The coverage circles about each station have a radius of $1140 \mathrm{~km}$, corresponding to an elevation of $15^{\circ}$ and an ionospheric pierce point altitude of $400 \mathrm{~km}$. Also shown are the magnetic equator and the lines of magnetic dip angle.

where $W(x)$ is the Lambert $W$ function. Figure 2a displays the probability density distribution of $p$ as measured by the ISM and we note that the result is not physical. Values in the range -1 to 1.5 are typically obtained not 2.5 to 3 as expected. However, in Fig. $2 \mathrm{~b}$ we observe that the values of $p$ calculated via Eq. 3 are in the expected range and as such we have confidence in these values. Thus, in what follows, we use $p$ as calculated by Eq. 3 .

\section{Phase screen theory}

To proceed we are required to convert the scintillation data to a parameter which describes the strength of the irregularities. The strength of the turbulence or scattering structure, $C_{S}$, has been used to describe the irregularity strength and is given by (Rumsey, 1975; Rino, 1979):

$$
C_{s}=8 \pi^{(3 / 2)} \frac{\Gamma(v+1 / 2)}{\Gamma(v-1)} q_{o}^{2 v-2}\left\langle\Delta N^{2}\right\rangle,
$$

where $v \approx p / 2$. The phase spectral slope is related to the irregularity spatial spectral index, $q$, by $p=q+1 .\left\langle\Delta N^{2}\right\rangle$ is the variance of the electron density, and $q_{o}$ the outer scale size of the irregularities. $C_{s}$ is equal to the strength of the 3-D spatial spectrum of the irregularities at a wave number of $1 \mathrm{rad} \mathrm{m}^{-1}$.
It has been shown by Rino and Fremouw (1977) and Rino (1979) that if the irregularities are assumed to act as a single scatter thin diffracting phase screen then by applying the Born approximation the following relation between $S_{4}$ and $C_{s}$ may be derived:

$S_{4 w}^{2}=r_{e}^{2} \lambda^{2} C_{s} L \frac{\sec \theta F}{2 \sqrt{\pi}}\left[\frac{\Gamma\left(\frac{2.5-v}{2}\right)}{\Gamma\left(\frac{v+0.5}{2}\right)(v-0.5)}\right] Z^{v-0.5}$

where $r_{e}$ is the classical electron radius, $\lambda$ the wavelength, $L$ the layer thickness, $\theta$ the incident angle on the phase screen, $F$ the Fresnel filter term, and $Z$ the Fresnel zone parameter. The $w$ subscript on $S_{4}$ in Eq. 5 denotes that this is $S_{4}$ due to the single scatter of the wave field. In a multiple scattering regime $S_{4}$ may become saturated and this is accounted for by Rice statistics:

$S_{4}^{2} \approx 1-\exp \left(-S_{4 w}^{2}\right)$.

The Fresnel filter term, $F$, is dependent on propagation and the shape and orientation of the irregularities (for details see Rino, 1979; Fremouw, 1980). The Fresnel zone parameter is given by

$Z=\frac{\lambda z_{r} \sec \theta}{4 \pi}$, 

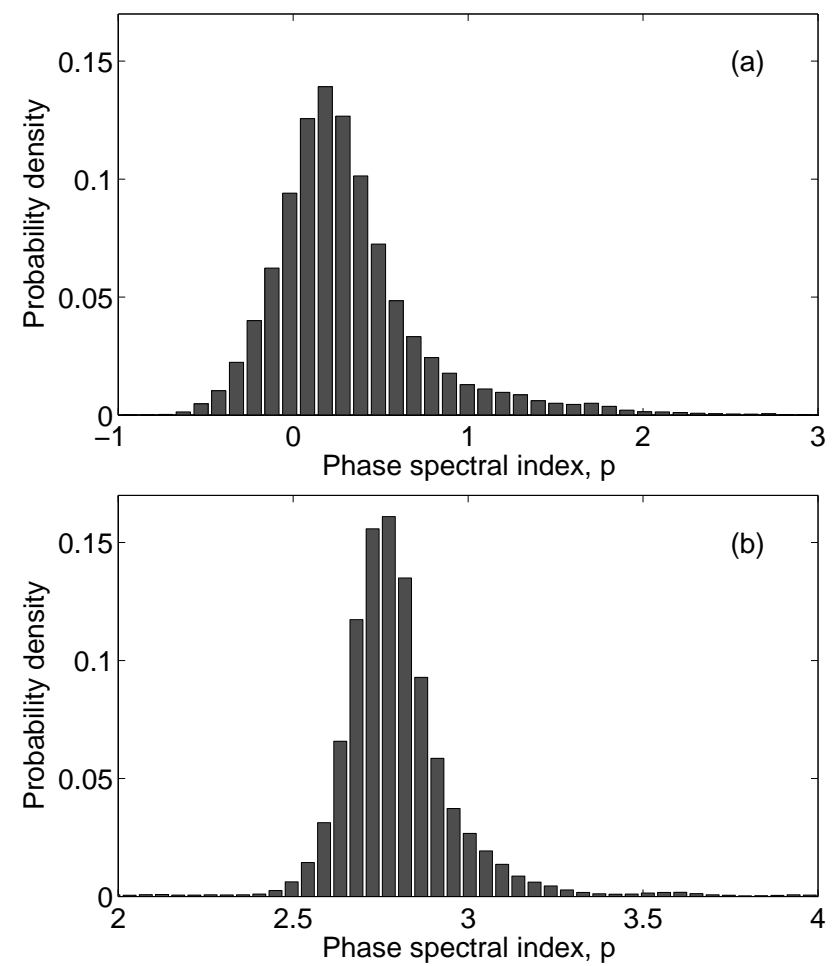

Fig. 2. Probability density distribution of $p$ as measured by the ISM (a) and as calculated by Eq. 3 (b). The mode of the distribution in panel (b) is 2.75 and the median is 2.77. Data are from September 2000 .

where $z_{r}$ is the reduced height:

$z_{r}=\frac{z_{1} z_{2}}{z_{1}+z_{2}}$

with $z_{1}$ and $z_{2}$ the vertical distances from the observer and source to the phase screen

An expression equivalent to Eq. (5) which relates the phase spectral strength $T$ to $C_{s}$ may also be derived:

$T=r_{e}^{2} \lambda^{2} C_{s} L G \sec \theta\left[\frac{\sqrt{\pi} \Gamma(v)}{(2 \pi)^{2 v+1} \Gamma(v+0.5)}\right] V_{e}^{2 v-1}$,

where $G$ is a geometrical parameter which like $F$ is dependent on the geometry of the irregularities. The quantity $V_{e}$ is the effective velocity of the irregularities which is the scan velocity modified to account for the effect that the anisotropy of the irregularities has on the conversion from the spatial (structure) to temporal (scintillation) domain. The scan velocity is the velocity relative to the irregularities of the point where the radio path cuts the phase screen. Thus, $V_{e}$ is dependent on the drift velocity of the irregularities, the velocity of receiver and transmitter, and the shape and orientation of the irregularities.

Previous researchers have used the height integrated irregularity strength defined at a scale size of $1 \mathrm{~km}, C_{k} L$, to de- scribe the irregularities (e.g. Secan et al., 1995) and it is related to $C_{s}$ as follows:

$C_{k} L=1000^{(2 v+1)} C_{s} L$.

This formulation has the advantage that the layer thickness is not required to be known. We follow this convention.

\section{Data processing}

The theory outlined above requires us to assume a height at which the phase screen is to be placed. Previous researchers (Rino and Fremouw, 1977; Rino, 1979; Fremouw, 1980; Secan et al., 1995) have used $350 \mathrm{~km}$ and we also use this height. The shape and orientation of the irregularities is also required; we have assumed field aligned rods. Finally we note that the disadvantage of this theory is that information about the vertical structure of the irregularities is not obtained.

A $15^{\circ}$ elevation mask is first applied to the 1 minute resolution $S_{4}$ data and then $C_{k} L$ calculated from Eq. (5), (6) and (10) in conjunction with Eq. 3). We do not use Eq. (9) to calculate an alternative value for $C_{k} L$ as we have no way of measuring the drift speed of the irregularities, hence $V_{e}$ is unknown. For each resulting datum, the geographical latitude and longitude are calculated (assuming spherical Earth geometry) based on the recorded elevation and azimuth of the satellite and the height of the phase screen, and may be regarded as the position of the ionospheric penetration point (IPP) of the satellite-receiver link. The local solar time at the IPP is calculated for each datum from the recorded UTC time stamp.

$C_{k} L$ data from each month are binned according to the local solar time $(1 / 2 \mathrm{~h}$ bins) and geomagnetic dip angle ( 2 degree bins) at the IPP. We use magnetic dip rather than magnetic latitude as dip is an actual magnetic field quantity whereas magnetic latitude relies on a model (e.g. offset dipole) to define it. As the irregularities are geomagnetically controlled we consider that it is better to use the real field (i.e. magnetic dip) to bin the data. For reference, in the South-East Asian region, near the anomaly crests, the magnetic latitude is approximately half the dip angle. The slant $S_{4}$ data used to calculate $C_{k} L$ may also be binned in this manner which allows us to examine exactly where the scintillation activity is observed to originate from. Figure 3 displays the the upper decile slant $S_{4}$ index at Pare Pare during September 2000. The peak activity is equator-ward of the anomaly crest which is not surprising given that poleward of the anomaly the scintillation activity drops off rapidly.

Figure 4 displays upper decile $C_{k} L$ observed from Pare Pare during September 2000 using calculated values (top) and a fixed value (bottom) for $p$. The mode of the probability density distribution for $p$ (2.75) was chosen for the fixed value. We discuss this result in detail in the next section. Here we limit the discussion to two points. The first thing 


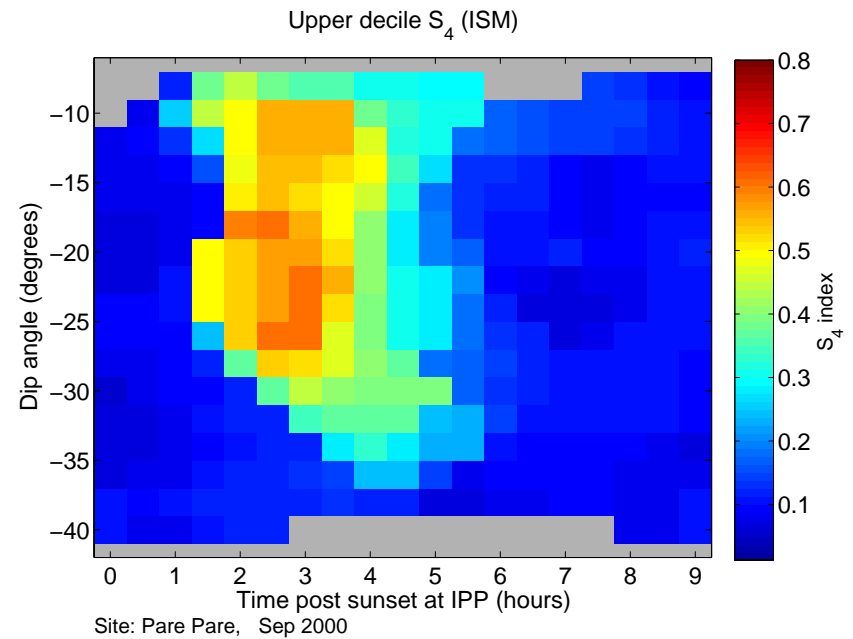

Fig. 3. Upper decile slant $\mathrm{S}_{4}$ index at Pare Pare during September 2000 .

to note is the effect of the geometrical corrections on the geographical distribution of the data vis-à-vis Fig. 3: the peak has moved southward as expected. Secondly a comparison of the top and bottom panels shows that there are only minor differences between the two results. Thus, we conclude that when the phase data are unusable (e.g. due to contamination by noise), the employment of a fixed representative value for $p$ is a viable alternative.

We have assumed that there is a high degree of correlation of scintillation occurrence over longitude, not only across the field of view at each site, but also over the region in general. This is validated by the results of Thomas et al. (2001) and is due to the very small magnetic declination angle over the region.

\section{Results}

We present results from two equinoctial sunspot maximum months: September 2000 (Fig. 5) and March 2001 (Fig. 6). In each figure upper decile $C_{k} L$ is displayed as a function of time post sunset, $t_{\mathrm{pss}}$, at the IPP and geomagnetic dip angle for the 3 aforementioned sites. All the results displayed here were produced using $p$ calculated from Eq. (3).

The following points are noted:

1. Gross features are as expected with peaks in the activity near the anomaly crests and a reduction at the magnetic equator.

2. Irregularity activity starts at $t_{p s s} \approx 1.5-2 \mathrm{~h}$ and finishes at $t_{p s s} \approx 5.5-7 \mathrm{~h}$, depending on site and season.

3. Peak activity occurs at magnetic dips of $\approx-27^{\circ}$ and $+27^{\circ}$ (geomagnetic latitudes $-12.5^{\circ}$ and $+12.5^{\circ}$; geographic latitudes $-4.3^{\circ}$ and $+20.3^{\circ}$ ).

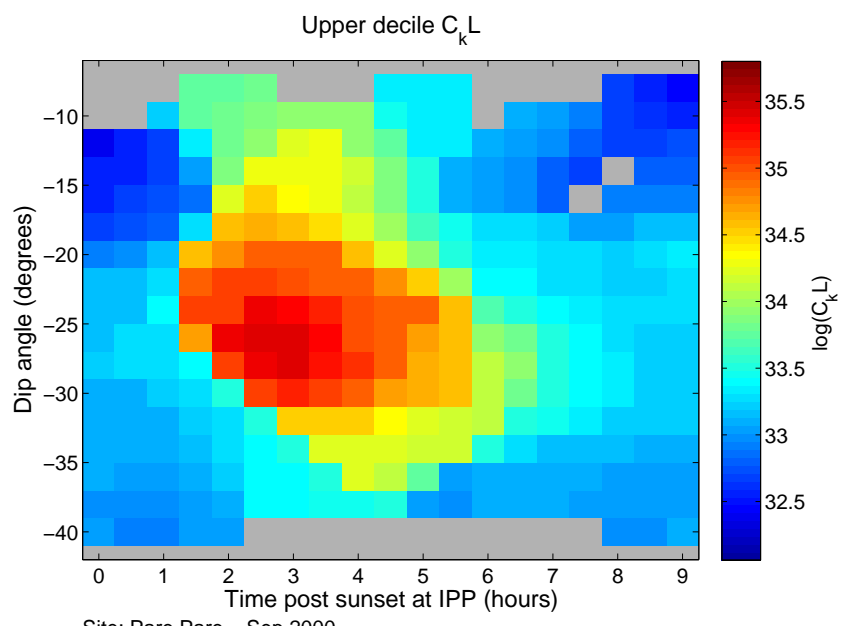

Site: Pare Pare, Sep 2000

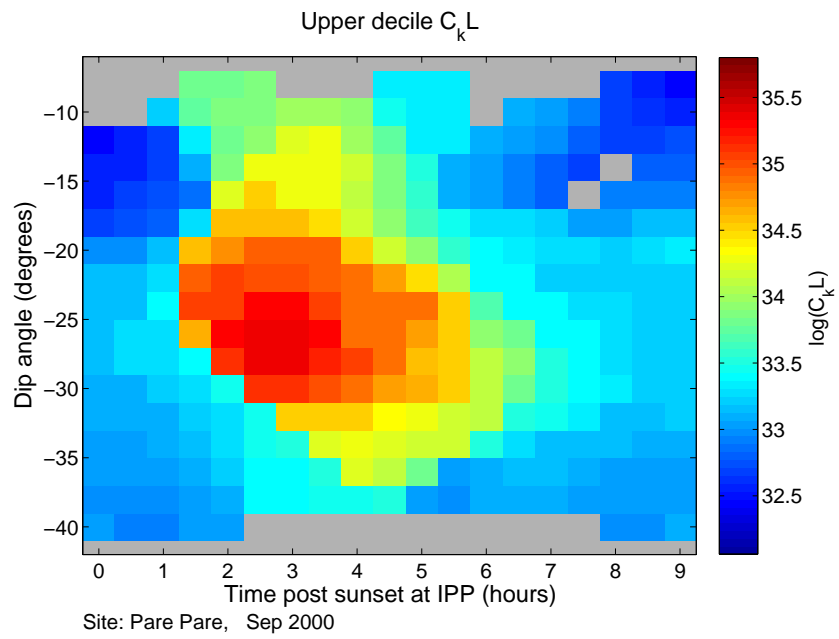

Fig. 4. Upper decile $C_{k} L$, September 2000, at Pare Pare. The top panel displays results using $p$ calculated from Eq. (3) while the bottom panel a fixed value for $p$ of 2.75

4. The irregularities at the northern anomaly (NA) persist longer (about 1-1.5 h) into the night than at the southern anomaly (SA) during the September equinox.

5. During the March equinox, the northern anomaly activity cuts off $\sim 1.0 \mathrm{~h}$ earlier than during September. This behaviour is not noted at the southern anomaly.

6. Southern and northern anomaly activity as a function of $t_{\mathrm{pss}}$ differ with the southern anomaly activity occurring at higher latitudes with increasing $t_{\mathrm{pss}}$. This behaviour is not noted at the northern anomaly. It is observed at both the vernal and autumnal equinoxes.

7. The Marak Parak results are inconsistent with the SA and NA station results where they overlap. In addition a single peak is observed near the magnetic equator rather than an expected trough with increasing activity with increasing latitude north and south. 

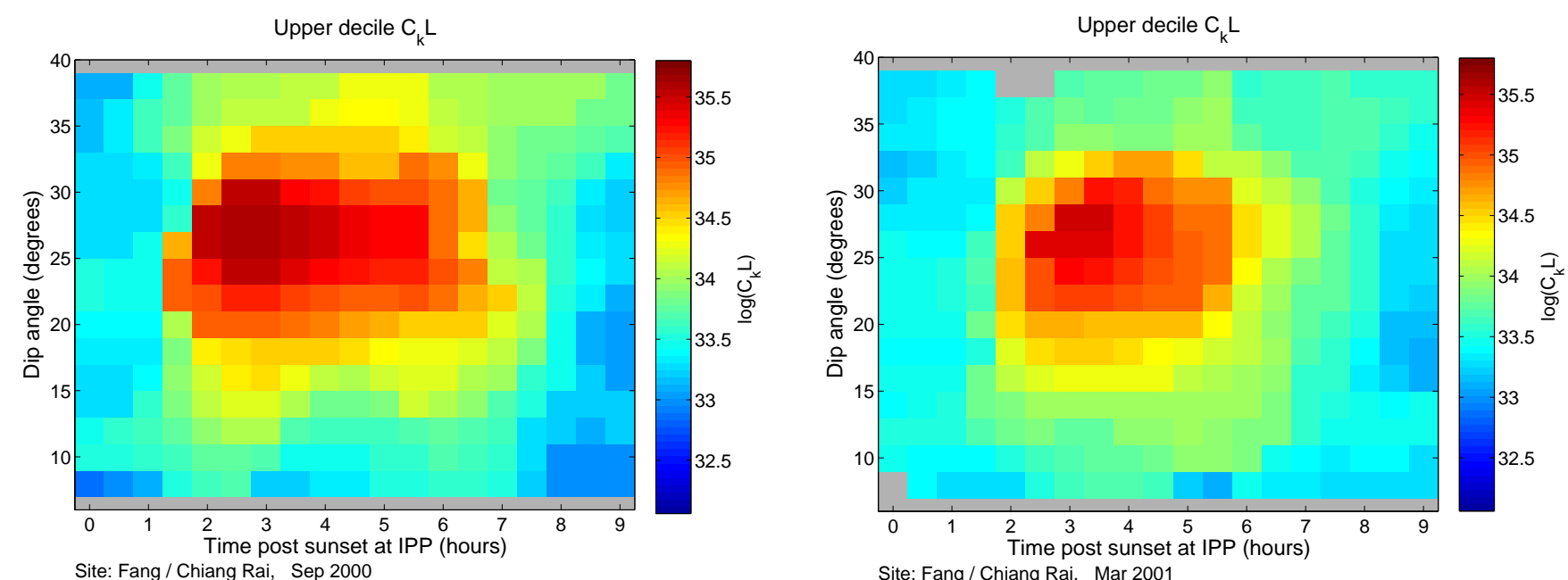

Upper decile $C_{k} L$

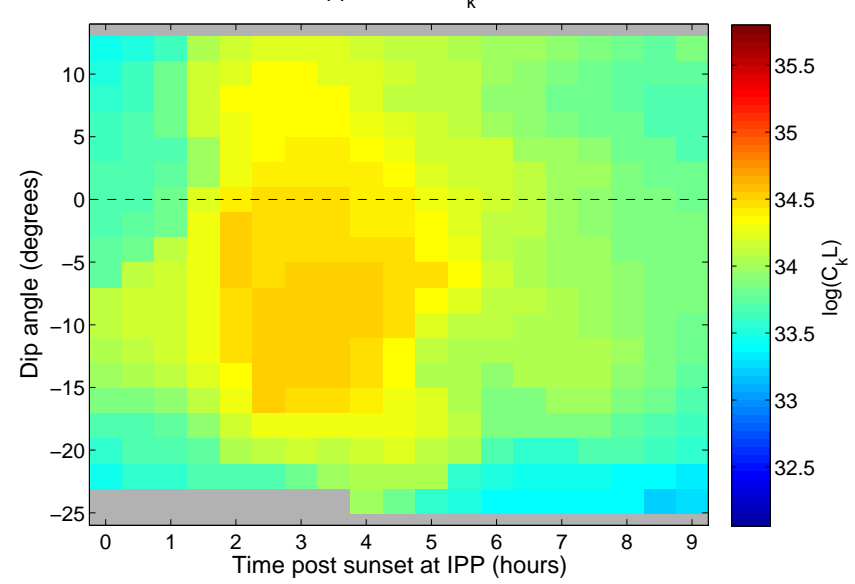

Site: Marak Parak, Sep 2000

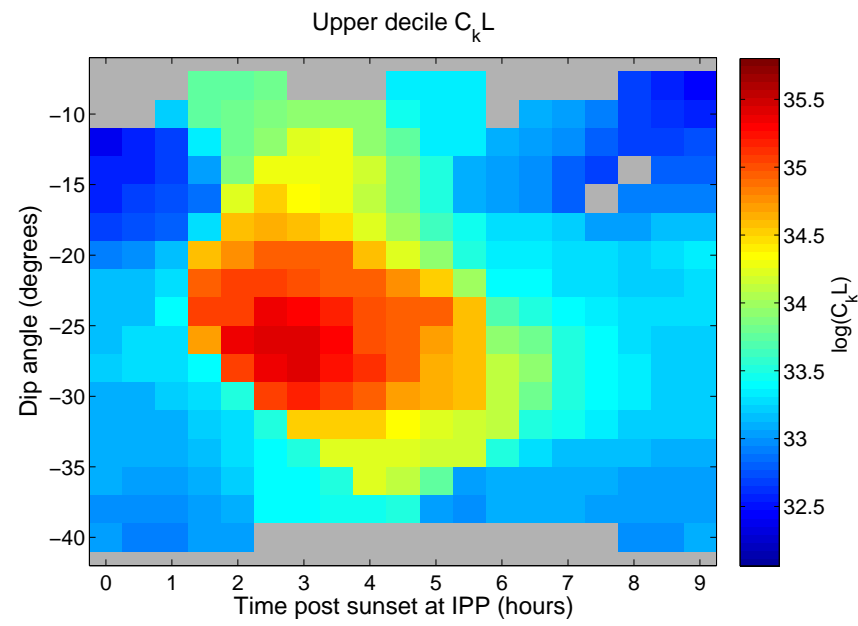

Site: Pare Pare, Sep 2000

Fig. 5. Upper decile $C_{k} L$, September 2000, at Chiang-Rai (top), Marak Parak (middle) and Pare Pare (bottom). The dashed line indicates the magnetic equator.

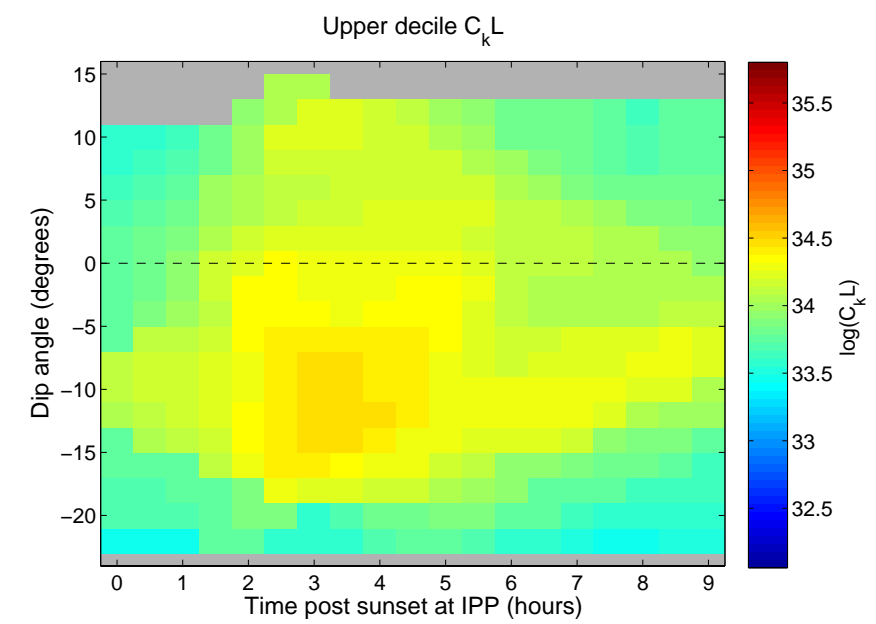

Site: Marak Parak, Mar 2001

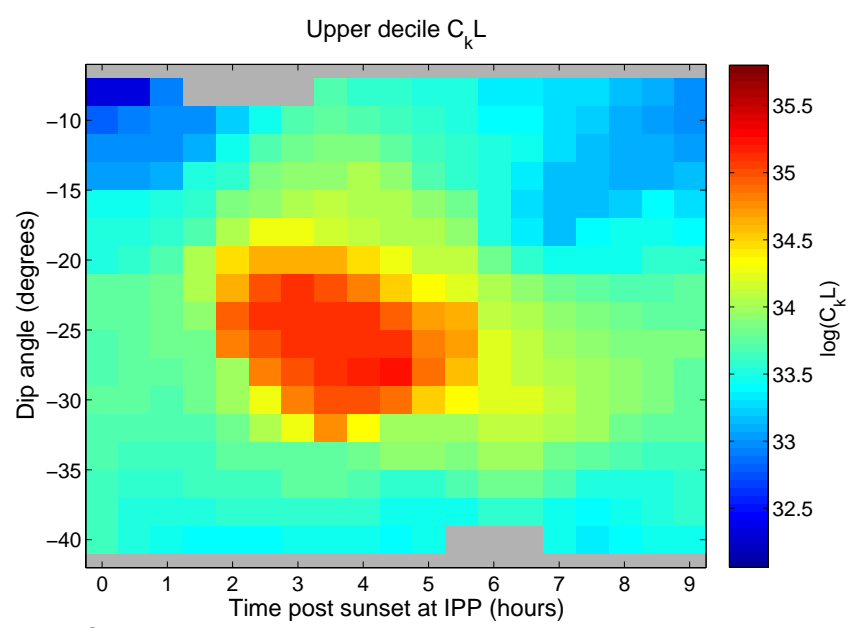

Site: Pare Pare, Mar 2001

Fig. 6. Upper decile $C_{k} L$, March 2001, at Chiang-Rai (top), Marak Parak (middle) and Pare Pare (bottom). The dashed line indicates the magnetic equator. 
We discuss these points below.

\subsection{Latitudinal position of irregularity activity}

While the first two points above are expected from the known climatology of scintillation at L-band frequencies (Aarons and Basu, 1994; Basu et al., 1980), the third point, at first impression, seems to indicate that the peak activity is biased equator-ward from the anomaly crests by $2^{\circ}-3^{\circ}$ in magnetic latitude. However, this is not the case as the anomaly crests move equator-ward after the passage of the sunset terminator as displayed in Fig. 7. This figure displays $\mathrm{f}_{\mathrm{o}} \mathrm{F} 2$ generated by the International Reference Ionosphere (IRI90) (Bilitza, 1990). Clearly, not only does the strength of the anomalies decrease post sunset, they also move equator-ward. In fact at the time when activity level of the irregularities maximises ( 20:30 LT), the latitude of the peaks in irregularity activity and the background ionosphere coincide at the northern anomaly while at the southern anomaly the background ionosphere is even further equator-ward than our results. We do not infer anything from the latter point as the ionosphere used in the comparison is that from a model (albeit empirically derived from real data) rather than a real ionosphere.

The reason for the anomaly crests moving equator-ward post sunset is probably due to recombination processes preventing the vertically moving plasma at the magnetic equator from reaching as high an altitude than during the day. Thus, when the plasma (and also the irregularities) map down the magnetic field lines they do not travel as far from the equator as it would otherwise do during the day.

\subsection{Variability of irregularity temporal behaviour}

The behaviour noted by points 4 and 5 could be due to variability of either (1) the irregularity seeding mechanism or (2) the background ionosphere itself. We consider each of these possibilities in turn.

\subsubsection{Temporal variability of irregularity seeding}

Tropical convection is one of the most important mechanisms for the generation of non-orographic gravity waves (Dhaka et al., 2001, 2003) which may subsequently seed ionospheric irregularities when they propagate up to the $\mathrm{F}$ region. Vincent (private communication, 2005) indicates that gravity waves with horizontal scales of $400 \mathrm{~km}$ are important for the seeding of irregularities in the ionosphere and they take $\sim 1 \mathrm{~h}$ to propagate from the ground to ionospheric heights. The observations of Kelley et al. (1981) and Nicolls and Kelley (2005) show strong evidence for the seeding of ionospheric irregularities by gravity waves with horizontal scales of $\sim 600 \mathrm{~km}$.

The irregularity generating region (which is at the magnetic equator) for the Chiang Rai (north anomaly) data is over land near a moderately large mountain in southern Thailand (Khao Luang, $1786 \mathrm{~m}$ ). However, the irregularity generating region for the Pare Pare (south anomaly) data is in

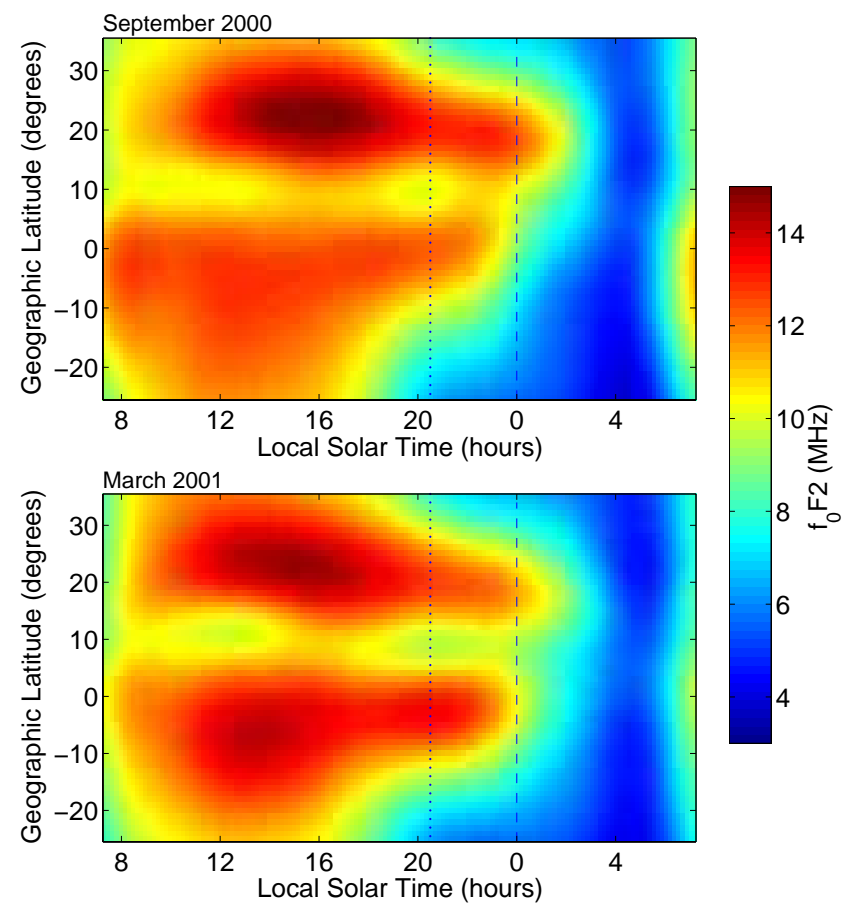

Fig. 7. Critical frequency of the $\mathrm{F} 2$ layer at the $110^{\circ}$ meridian as a function of time and geographic latitude generated by IRI90. The dotted line indicates the time when the peak irregularity activity occurs while the dashed line indicates local midnight

the Sulu Sea. Atmospheric convection is stronger over land than sea (Soden, 2000; Yang and Slingo, 2001; Tian et al., 2004). Furthermore, continental convection peaks in the early evening ( $\sim$ 17:00-22:00 LST) whereas oceanic convection peaks in the early morning ( 06:00-09:00 LST). This could explain the observed later cutoff of ionospheric irregularities at Chiang Rai than at Pare Pare during the September equinox; the convection induced gravity waves would occur later into the night over land than over sea. Figure 8 displays the mean deep convection activity (DCA) index for the two irregularity generating regions during March and September. The DCA was derived from the Cloud Archive User Service (CLAUS) archive of global brightness temperatures from 1989 to 1991 employing the same methodology as Yang and Slingo (2001). The CLAUS archive is based on the International Satellite Cloud Climatology Project (ISCCP) level B3 data (Rossow et al., 1997). We acknowledge that the CLAUS data is not contemporary with our scintillation data. However, as we are only examining climatology we feel this is not invalid given that the data are from similar periods in the solar cycle. Figure 8 shows a strong peak in the post sunset convection activity at the Chiang-Rai irregularity generation region during September which is absent in the Pare Pare generation region. This is consistent with the discussion in the previous paragraph. During the March equinox low convection activity in both the regions of interest is ob- 

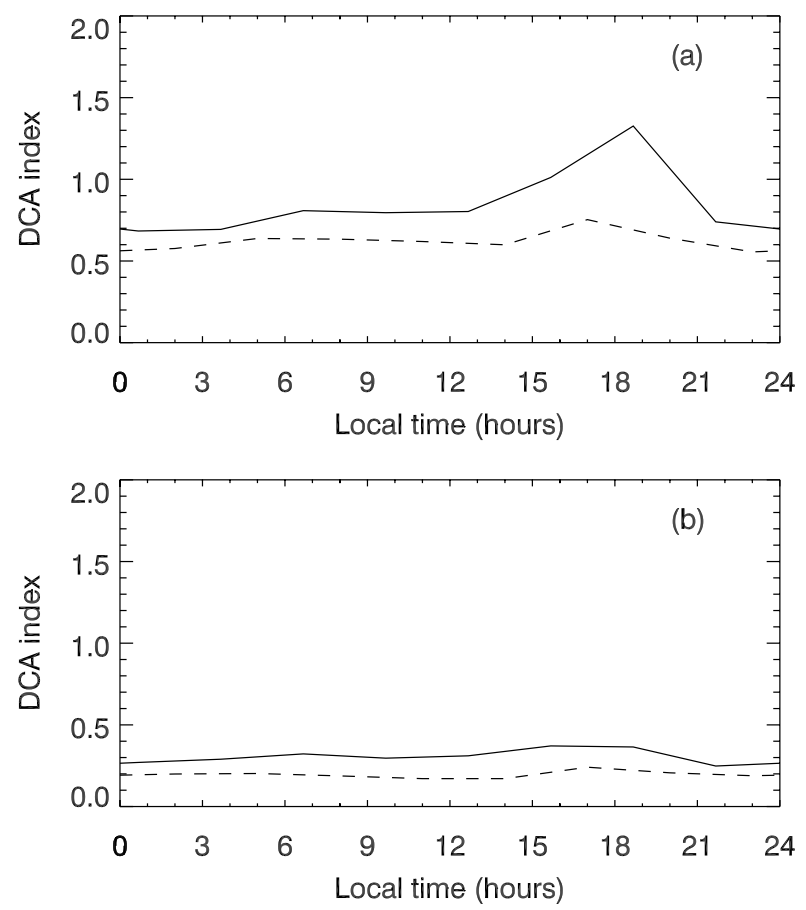

Fig. 8. Mean deep convection activity (DCA) index at (a) the Chiang-Rai and (b) the Pare Pare irregularity generating regions for September (solid line) and March (dashed line).

served and this may be a possible explanation of the lack of late scintillation activity at either of sites.

5.2.2 Temporal variability of the anomalies' cessation times

We now consider the latter mechanism: variability of the background ionosphere. Figure 7 shows that IRI90 indicates the northern anomaly holds up well past local midnight and $\sim 2 \mathrm{~h}$ later into the night than the southern anomaly during the September equinox. However, during March this discrepancy is much reduced with the southern anomaly lasting longer than during September while the northern anomaly finishes earlier. While IRI is only a model, it is empirically based and as such it captures the climatological behaviour of the ionosphere. The variability of the ionosphere as modeled by IRI at the anomalies during the two equinoxes is in line with the noted irregularity durations of activity at both seasons and anomalies and thus is a possible explanation for it.

\subsubsection{Discussion}

Whilst acknowledging the caveats and the preliminary nature of the above analyses, we feel that both hypotheses presented here are plausible explanations for the variability of the observed irregularity temporal behaviour. Indeed it is likely that both mechanisms play a role. Further investigation is warranted, and to this end additional detailed observations of irregularity activity together with measurements of the background ionosphere, gravity wave activity and convection are required.

\subsection{Asymmetric irregularity behaviour at the anomalies}

The penultimate point is interesting and while we do not fully understand the cause of the different latitude vs. $t_{\mathrm{pss}}$ behaviour between the two anomaly regions, we posit the following possible explanation.

The neutral winds are the driving mechanism of the $S_{q}$ current system which gives rise to the eastward electric field in the $\mathrm{E}$ region. Over the geomagnetic equator the eastward conductivity takes the Cowling value due to the horizontal electric field and the vertical limitation of the conducting layer. Consequently the so-called equatorial electrojet is manifest and it in turn a causes an enhanced eastward electric field. This E-region neutral wind dynamo, which drives the zonal eastward electric field, gives rise to a vertical $\boldsymbol{E} \times \boldsymbol{B}$ drift. Away from the geomagnetic equator, the Hall current leakage means that the eastward conductivity rapidly drops to the Pedersen value and thus the eastward electric field is much weaker. Consequently the $\boldsymbol{E} \times \boldsymbol{B}$ drift is much weaker and in addition is no longer vertical. However, it is not unreasonable to assume that it would still affect the motion of the plasma and irregularities to a small degree as they propagate down the field lines. The neutral winds are a moderator of the strength of the eastward electric field and as they are not symmetrical about the magnetic equator any residual $\boldsymbol{E} \times \boldsymbol{B}$ drift affecting the motion of plasma and irregularities will also not be symmetrical. This may give rise to different behaviour at the two anomalies.

In order to verify our hypothesis detailed observations of the diurnal variation of the zonal electric field as well as the dynamics of the neutral wind at several latitudes in the region are required.

\subsection{Limitations of thin phase screen theory}

The last point we listed at the start of this section is important and we believe it is an indicator of the limitations of applying thin phase screen theory to represent irregularities which have a vertical structure. Physical models of irregularity formation (e.g. Keskinen et al., 2003) clearly show that thin phase screen models need to be updated.

Figure 9 displays the upper decile slant $\mathrm{S}_{4}$ at Marak Parak during September 2000. We observe, as expected, that the scintillation activity is concentrated away from the geomagnetic equator where a trough in the activity level is known to occur.

When the theory is applied to calculate $C_{k} L$ we expect the activity level to rise at the geomagnetic equator (i.e. the trough become shallower) as the geometrical effects on the 


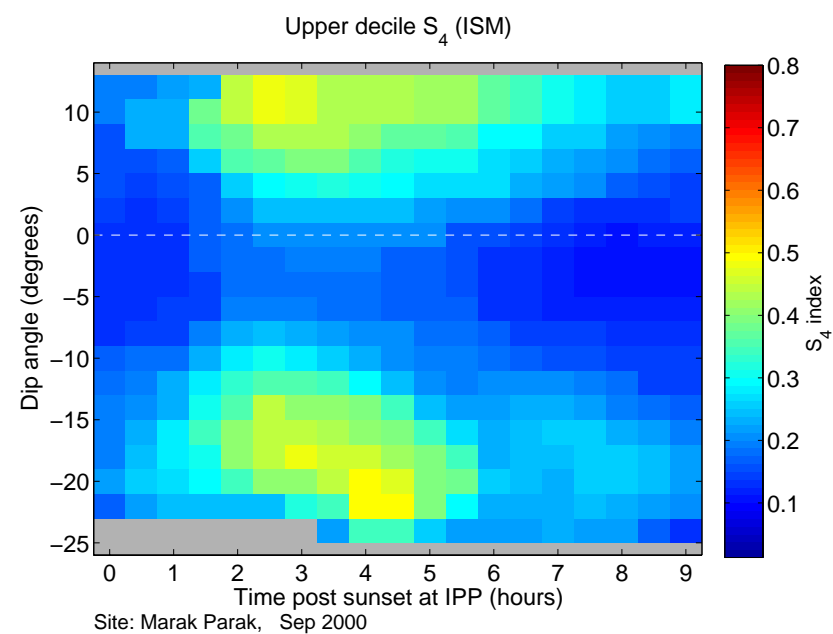

Fig. 9. Upper decile slant $\mathrm{S}_{4}$ index at Marak Parak during September 2000. The dashed line indicates the magnetic equator.

large off-zenith angle paths are corrected. However, it is clear from a comparison of Fig. 9 with the Marak Parak $C_{k} L$ data displayed in Fig. 5 that the theory has over-compensated for the geometrical effects and furthermore the over-correction becomes progressively worse with increasing off zenith angle.

\subsubsection{Large off-zenith angle issues}

The quantities from Eq. (5) which affect $C_{k} L$ as a function of ray elevation are the Fresnel filter factor, $F$, and $Z \sec (\theta)$. Figure 10 (top) displays $F$ as a function of ray elevation at Marak Parak. Examination of this figure shows that peaks occur at $\sim 12^{\circ}$ South and $\sim 10^{\circ}$ North where the rays are perpendicular to the magnetic field. At these ray elevations the reduction factor applied to $C_{k} L$ is a maximum. However, the variation of $F$ with ray elevation is not great and it is not enough to explain the observed drop in $C_{k} L$ activity for low elevation rays.

In Fig. 10 (bottom) we examine $Z \sec (\theta)$. At high elevations the reduction factor is small e.g. at $45^{\circ}$ (assuming all else is equal) $C_{k} L$ is reduced to $74.6 \%$ of its zenithal value. However, as the elevation decreases to $30^{\circ}$ and further to $15^{\circ}$ the effect on the calculated $C_{k} L$ is to reduce it to $17.6 \%$ and $1.9 \%$ respectively. Clearly $Z \sec (\theta)$ has a large effect on low elevation rays and is the main reason for the calculated low $C_{k} L$ activity at low elevations at Marak Parak. However, $Z \sec (\theta)$ is site independent, thus the question is raised why similar behaviour at Pare Pare and Chiang-Rai is not observed. Examination of Fig. 11 helps to resolve this question. In this figure we see that whilst at Marak Parak the low elevation $\left(15^{\circ}\right)$ rays to the North and South traverse the anomaly regions with concomitant high levels of scintillation (see Fig. 9), at Pare Pare these rays miss the southern anomaly region. The south bound ray traverses the iono-
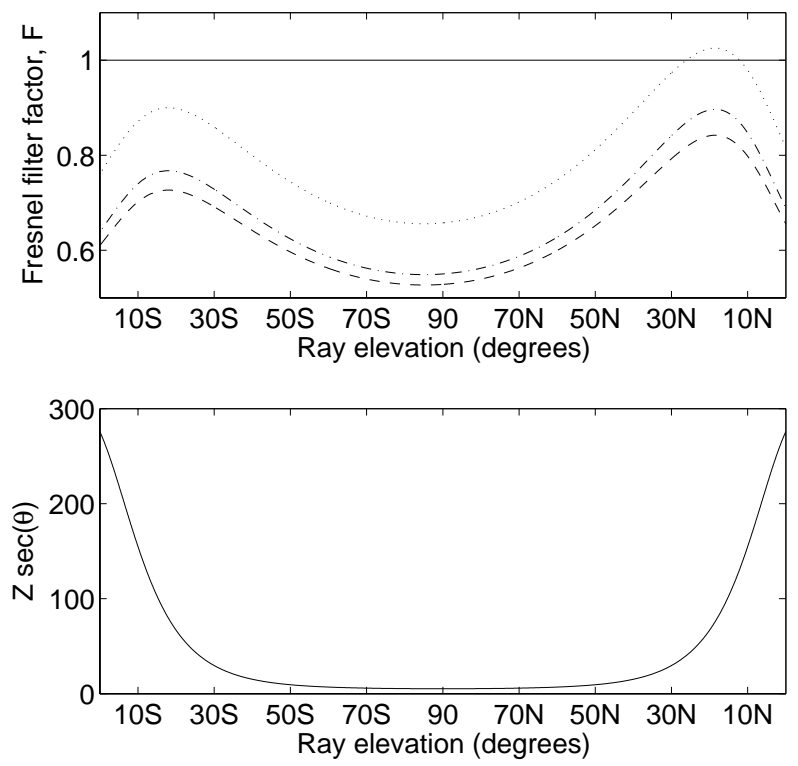

Fig. 10. Fresnel filter factor (top) and $Z \sec (\theta)$ (bottom) as a function of ray elevation at Marak Parak. The ray azimuth was fixed at either $0^{\circ}$ or $180^{\circ}$ and is indicated by the ordinate tick suffixes ("N" or "S" respectively). $F$ has been calculated for isotropic irregularities (solid line) and field aligned rods with axial ratios (parallel to the magnetic field) of 2 (dotted), 5 (dashed-dot) and 50 (dashed).

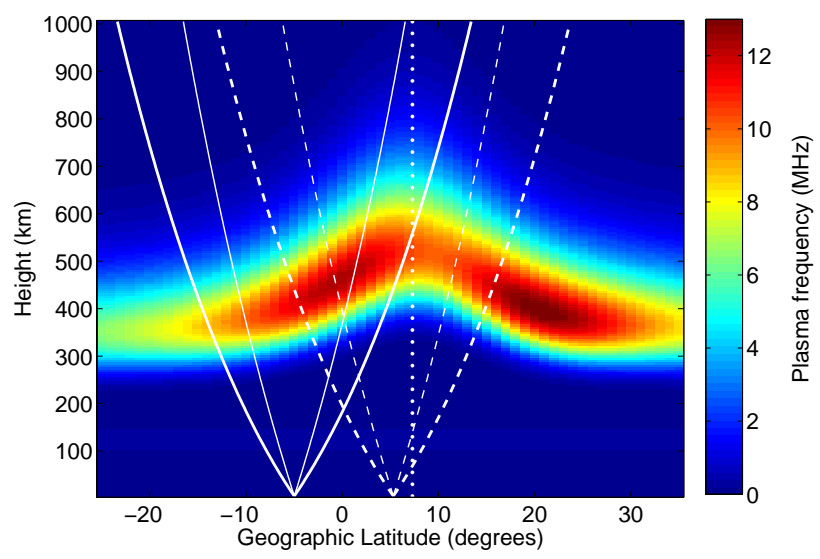

Fig. 11. IRI90 derived plasma frequency as a function of geographic latitude and height. The solid lines represent north and south bound rays with elevations of $15^{\circ}$ (thick) and $30^{\circ}$ (thin) originating from Pare Pare while the dashed lines are similar rays originating from Marak Parak. The ray paths appear curved due to the representation of curved Earth geometry in a rectilinear coordinate system. The dotted line indicates the geomagnetic equator.

sphere so far poleward of the anomaly that scintillation is not observed (see Fig. 3) while the north bound ray traverses the ionosphere near the magnetic equator where scintillation is much reduced in severity. The effect of $Z \sec (\theta)$ on $C_{k} L$ calculated from these rays is either non-existent (south bound 


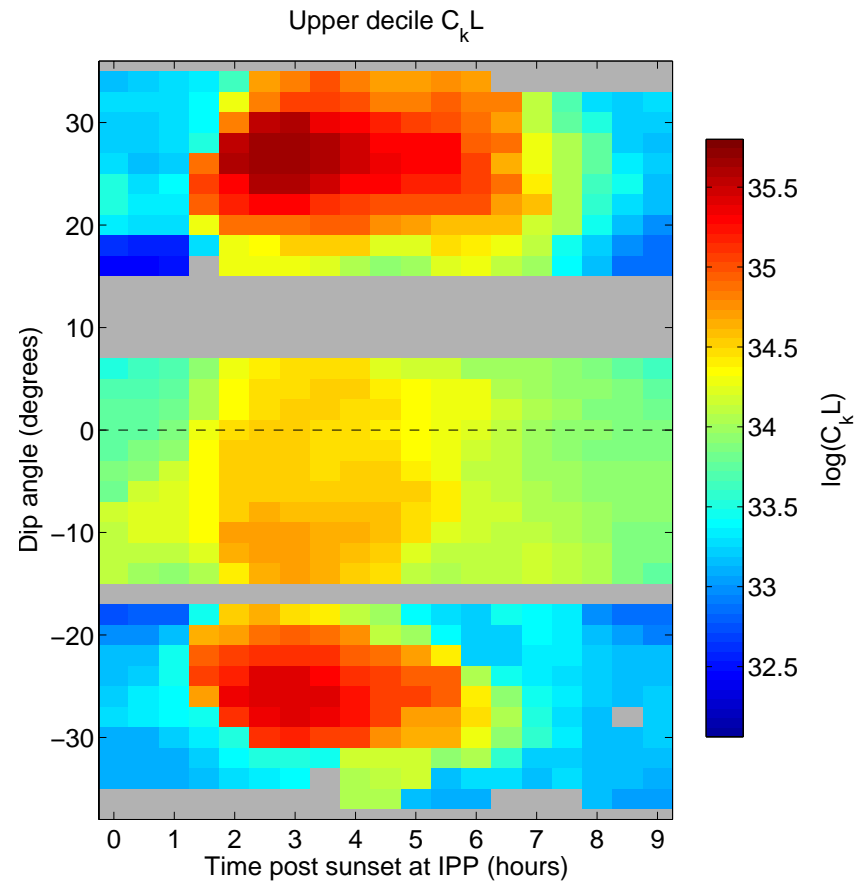

Fig. 12. Upper decile $C_{k} L$ combined from three sites during September 2000. Dashed line indicates magnetic equator. A $30^{\circ}$ elevation mask was applied to the raw data.

ray) or simply enhances the drop in activity near the equator (north bound ray). Thus, the effect is not obvious at Pare Pare where it is in line with the existing latitudinal variation unlike at Marak Parak. We have not displayed rays from ChiangRai in Fig. 11, however, from symmetry the same argument applies.

Turning our attention to the $30^{\circ}$ rays in Fig. 11 we observe that the north bound ray from Pare Pare intersects the south bound ray from Marak Parak near the height of the phase screen. It is interesting to note that $C_{k} L$ data derived from these rays (occurring at $\sim 16^{\circ} \mathrm{N}$ at Pare Pare and $\sim 15^{\circ} \mathrm{N}$ at Marak Parak) are in close agreement. This together with the much reduced effect of $Z \sec (\theta)$ at this elevation suggests that $30^{\circ}$ is a more appropriate elevation mask to apply to the data. Since WBMOD employs the same phase screen theory, these observations suggest that caution should be exercised when using this model for low elevation receiver-satellite links.

With the above in mind we reprocessed the September 2000 data using a $30^{\circ}$ elevation mask. As the $C_{k} L$ results for the three sites now do not overlap we display them as a combined plot in Fig. 12. We observe that while the situation has improved there are still problems at the interface of the Marak Parak and Pare Pare data. The southern most Marak Parak data holds up while the northern most Pare Pare data rapidly declines which together seemingly indicates an extra small peak in activity. Needless to say, we do not believe this and we investigate it below in Sect. 5.4.2 as a possible issue with the height of the phase screen.

Another interesting point to note is that the Northern Anomaly activity now extends further poleward. This is probably due to the exclusion of the low elevation data which had its calculated $C_{k} L$ activity suppressed by the overcorrection problem discussed above. Thus, we regard this plot to be a more accurate representation of irregularity activity.

\subsubsection{Phase screen height issues}

It is well known that the ionospheric height varies spatially to a large degree in the equatorial region and Fig. 11 displays this clearly with the ionosphere attaining its maximum height at the magnetic equator. Also apparent in this plot is that the $30^{\circ}$ elevation south-bound ray from Marak Parak traverses the southern anomaly region. Thus, the apparent anomalous peak noted in Fig. 12 is probably due to scintillation activity from the southern anomaly observed by the Marak Parak ISM which has been mapped to a latitude too far equator-ward. This incorrect mapping is due to the phase screen being placed at too low an altitude.

It is beyond the scope of this paper to include an exhaustive investigation on the phase screen height. However, we have repeated our analysis with a variable height phase screen. For each satellite-receiver link at every 1 minute epoch that we have data, the phase screen is placed at that height where the plasma density, as given by IRI90, is a maximum. There is now some overlap of the Marak Parak and Pare Pare data. In these regions the average value is taken. The result is displayed in Fig. 13 and we see an improvement in the latitudinal distribution of irregularity activity equator-ward of the anomaly regions. Figure 13 suggests that the use of a fixed phase screen height is not appropriate in the equatorial region and that models such as WBMOD, which rely on phase screens, might be improved by including the ability to use a variable height phase screen.

\subsection{Irregularity $C_{k} L$ probability density function}

Finally in Fig. 14 we examine the probability density function (PDF) of $C_{k} L$ at Pare Pare at the peak activity (dip $\left.=-27^{\circ}, t_{\mathrm{pss}}=3.0 \mathrm{~h}\right)$ and at two later times $\left(t_{\mathrm{pss}}=5.0 \mathrm{~h}\right.$ and $t_{\mathrm{pss}}=7.0 \mathrm{~h}$ ). Note the bi-modal nature of the PDF in Fig. 14a. This is as reported by Secan et al. (1995). However, later in Fig. 14b when the scintillation activity is lower, the strength of the upper peak is weaker relative to the lower peak. Finally, the upper peak is not in evidence at all in Fig. 14c when scintillation activity has finished. Thus, we interpret the lower peak of the PDF of our data as that due to noise, whether internal or external to the system, whereas the upper peak is interpreted as due to scintillation activity. The lower component reported by Secan et al. (1995) could be due to underlying irregularities at scale sizes which affect VHF and 


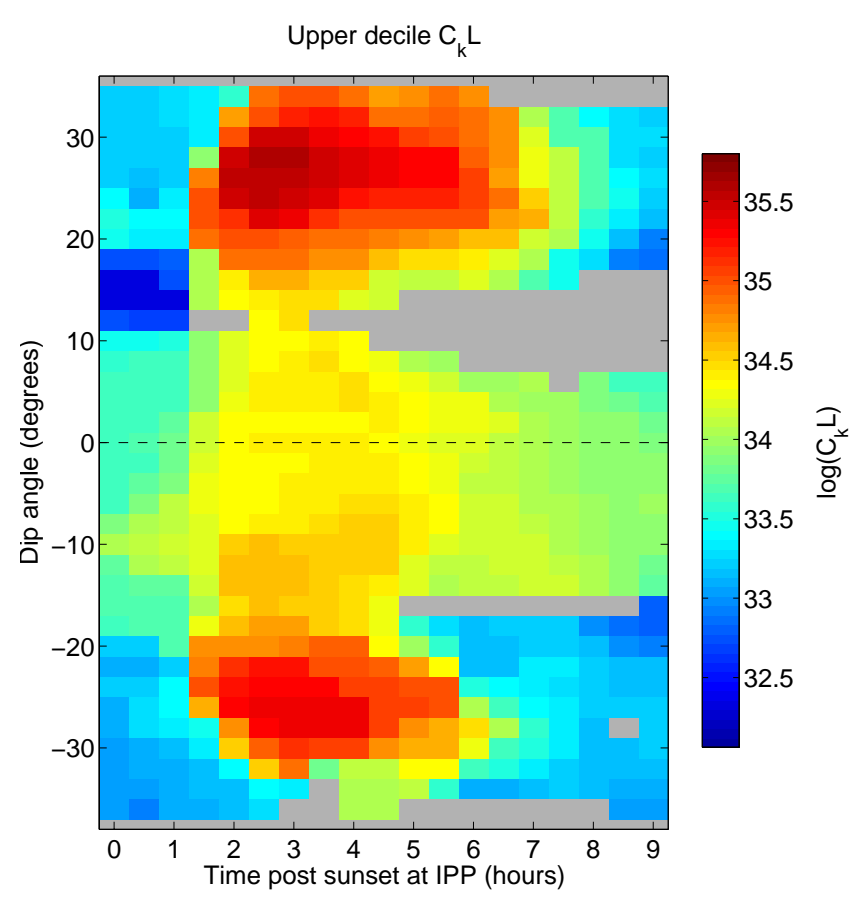

Fig. 13. As for Fig. 12 except here $C_{k} L$ was calculated using a variable height phase screen. See text for details.

UHF signals but do not develop sufficiently to have an effect on GPS signals at L-band.

When the phase screen height issues discussed in Sect. 5.4.2 have been fully resolved then the parametrisation of the $C_{k} L$ PDF as a function of magnetic dip angle, $t_{\mathrm{pss}}$, season and solar cycle will yield a climatological model of irregularities for the South-East Asian region.

\section{Conclusions}

Phase screen theory has been used to analyse scintillation on trans-ionospheric GPS signals. We have noted limitations of this technique: treating ionospheric irregularities as a thin phase screen ignores their inherent vertical structure and the calculated irregularity strength become less reliable with increasing ray-path off-zenith angle. In addition we noted there is some question as to a suitable phase screen altitude. Neverthe-less, with appropriate care, this technique yields useful insights into equatorial irregularity morphology.

We noted differences in the morphology of the irregularity activity at the two anomalies. Firstly, the activity duration varied with season. This could have been due to either seasonal variations in the background ionosphere or in the seeding mechanism. The latter was posited as being due to seasonal variations in tropical convection. Secondly, different latitudinal-temporal behaviour at the two anomaly regions was observed which we hypothesised as possibly being caused by the neutral winds generating eastward electric
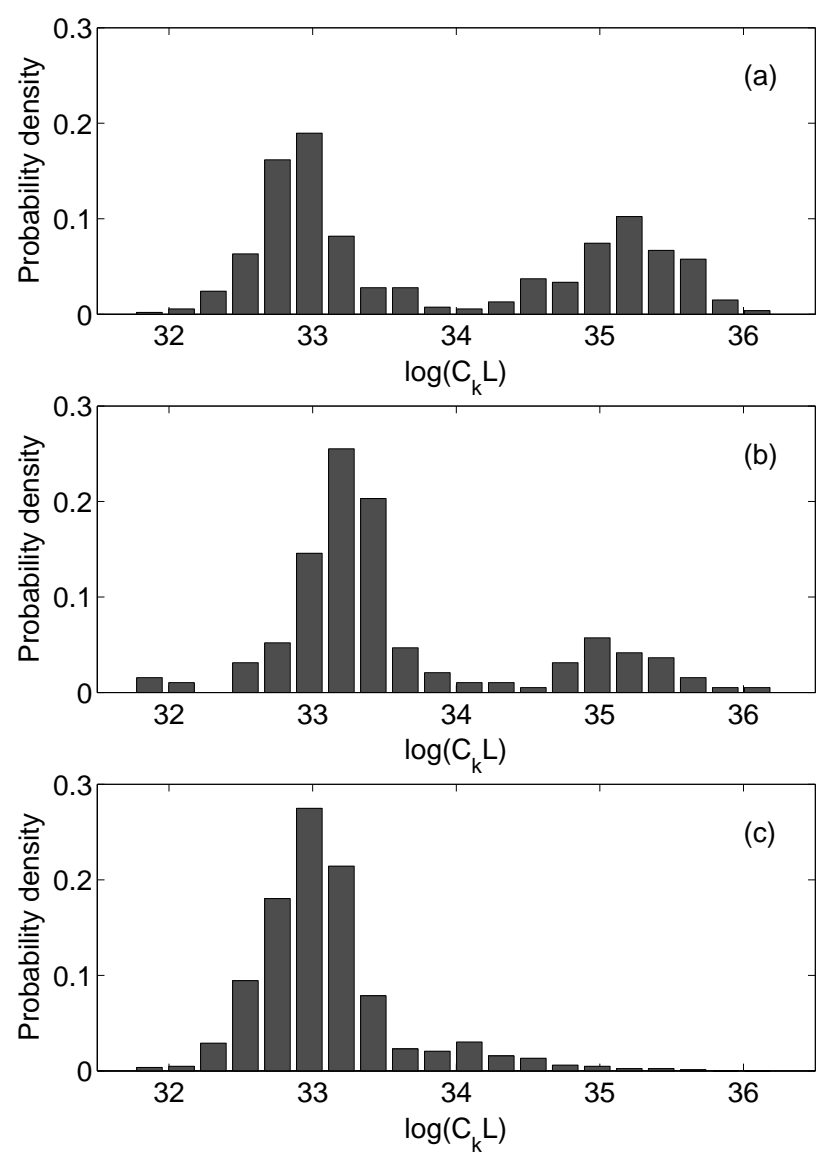

Fig. 14. $C_{k} L$ PDF from Pare Pare at dip $=-27^{\circ}$ and $t_{\mathrm{pss}}=3.0 \mathrm{~h}$ (a) and later at $t_{\mathrm{pss}}=5.0 \mathrm{~h}(\mathrm{~b})$ and $t_{\mathrm{pss}}=7.0 \mathrm{~h}(\mathrm{c})$.

fields asymmetric about the geomagnetic equator. Detailed observations of irregularity activity together with measurements of the background ionosphere, gravity wave activity, convection, zonal electric field and the dynamics of the neutral wind at several latitudes in the region are required to fully understand the morphology of equatorial irregularity activity and to confirm or reject the above theories.

We intend to use our data to climatologically characterise ionospheric irregularities in the South-East Asian region more precisely than has been hitherto achieved. This will allow models which rely on the irregularity strength to be improved in the region. Ultimately we require the ionospheric irregularities to be fully characterised in three dimensions. This will allow us to employ raytacing techniques to model the Doppler spread induced on HF signals (e.g. communications links, Doppler ionospheric sounders, HF radar) traversing the disturbed ionosphere (Coleman, 1996).

Other techniques such as tomography of the turbulent ionosphere (e.g. Kunitsyn and Tereshchenko, 1992b,a), or a multiple phase screen approach, may yield insights into the vertical structure of equatorial ionospheric irregularities. Alternatively, an improved scintillation theory which employs a 
3 dimensional structure function for the irregularities instead of the usual 2 dimensional form of thin phase screens may be required. The vertical profile of this 3-D structure function is as yet unknown and further observations and experiments with a more closely spaced chain of receivers together with 3-D physical models of irregularity formation (e.g. Keskinen et al., 2003) will be required to elucidate its form.

Acknowledgements. The deep convection activity results were obtained using the CLAUS archive held at the British Atmospheric Data Centre, produced using ISCCP source data distributed by the NASA Langley Data Center. The ISMs were loaned by K. Groves of AFRL, Hanscom Airforce Base, US. We thank A. G. bin Ramli of STRIDE, Malasia; Effendy of LAPAN, Indonesia; and P. Totorong of MRDC, Thailand for the provision of sites and data collection.

Topical Editor M. Pinnock thanks M. J. Keskinen for his help in evaluating this paper.

\section{References}

Aarons, J. and Basu, S.: Ionospheric amplitude and phase fluctuations at the GPS frequencies, in: Proceedings of the Institute of Navigation GPS-94, pp. 1596-1578, Institute of Navigation, Alexandria, Va., USA, 1994.

Basu, S., Basu, S., Mullen, J. P., and Bushby, A.: Long term $1.5 \mathrm{GHz}$ amplitude scintillation measurements at the magnetic equator, Geophys. Res. Lett., 7, 259-262, 1980.

Bilitza, D.: International Reference Ionosphere 1990, National Space Science Data Center (NSSDC) 90-22, Greenbelt, Maryland, 1990.

Cervera, M. A., Thomas, R. M., Ramli, A. G., Effendy, and Groves, K. M.: Validation of WBMOD in the South-East Asian Region, Radio Sci., 36, 1559-1572, 2001.

Coleman, C. J.: A model of HF sky wave radar clutter, Radio Sci., 31, 869-875, 1996.

Dhaka, S. K., Devrajan, P. K., Shibagaki, Y., Choudhary, R. K., and Fukao, S.: Indian MST radar observations of gravity wave activities associated with tropical convection, J. Atmos. Terr. Phys., 63, 1631-1642, 2001.

Dhaka, S. K., Takahashi, M., Kawatani, Y., Malik, S., Shibagaki, Y., and Fukao, S.: Observations of deep convective updrafts in tropical convection and their role in the generation of gravity waves, J. Met. Soc. Japan, 81, 1185-1199, 2003.

Fagundes, P. R., Sahai, Y., Batista, I. S., Abudu, M. A., Bittencourt, J. A., and Takahashi, H.: Observations of day-to-day variability in precursor signatures to equatorial F-region plasma depletions, Ann. Geophysicae, 17, 1053-1063, 1999.

Fremouw, E. J.: Geometrical control of the ratio of intensity and phase scintillation indicies, J. Atmos. Terr. Phys, 42, 775-782, 1980.

Huang, C. O., Kelley, M. C., and Hysell, D. L.: Nonlinear RayleighTaylor instabilities, atmospheric gravity waves and equatorial spread F, J. Geophys. Res., 98, 15 631-15 642, 1993.

Huang, C.-S. and Kelley, M.: Nonlinear evolution of equatorial spread-F: 1 . On the role of plasma instabilities and spatial resonance associated with gravity wave seeding, J. Geophys. Res., 101, 283-292, 1996a.
Huang, C.-S. and Kelley, M. C.: Nonlinear evolution of equatorial spread-F: 2. Gravity wave seeding of Rayleigh-Taylor insability, J. Geophys. Res., 101, 293-302, 1996 b.

Hysell, D. L., Kelley, M. C., Swartz, W. E., and Woodman, R. F.: Seeding and layering of equatorial spread $\mathrm{F}$ by gravity waves, J. Geophys. Res., 95, 17 253-17 260, 1990.

Kelley, M. C.: The Earth's Ionosphere, Academic Press, San Deigo, Calif., USA, p. 487 S., 1989.

Kelley, M. C. and Hysell, D. L.: Equatorial spread-F and neutral atmospheric turbulence: a review and comparative anatomy, J. Atmos. Terr. Phys., 53, 695-708, 1991.

Kelley, M. C., Larsen, M. F., LaHoz, C., and McClure, J. P.: Gravity wave initiation of equatorial spread F: A case study, J. Geophys. Res., 86, 9087-9100, 1981.

Keskinen, M. J., Ossakow, S. L., and Fejer, B. G.: Threedimensional nonlinear evolution of equatorial ionospheric spread-F, Geophys. Res. Lett., 30, 1855, 2003.

Kunitsyn, V. E. and Tereshchenko, E. D.: Radio tomography of the ionosphere, IEEE Antennas and Propagation, 34, 22-32, 1992a.

Kunitsyn, V. E. and Tereshchenko, E. D.: Determination of the turbulent spectrum in the ionosphere by a tomographic method, J. Atmos. Terr. Phys., 54, 1275-1282, 1992 b.

Nicolls, M. J. and Kelley, M. C.: Strong evidence for the gravity wave seeding of an ionospheric plasma instability, Geophys. Res. Lett., 32, L05 108, 2005.

Rino, C. L.: A power law phase screen model for ionospheric scintillation: 1. Weak scatter, Radio Sci., 14, 1135-1145, 1979.

Rino, C. L. and Fremouw, E. J.: The angle dependence of singly scattered wavefields, J. Atmos. Terr. Phys., 39, 859-868, 1977.

Rossow, W. B., Walker, A., and Roiter, M.: International Cloud Climatology Project (ISCCP): Description of reduced resolution radiance data, WTMO/TD-No. 58, 1997.

Rumsey, V. H.: Scintillations due to a concentrated layer with a power-law turbulence spectrum, Radio Sci,, 10, 107-, 1975.

Secan, J. A., Bussey, R. M., and Fremouw, E. J.: An improved model of equatorial scintillation, Radio Sci., 30, 607-617, 1995.

Singh, S., Johnson, F. S., and Power, R. A.: Gravity wave seeding of equatorial plasma bubbles, J. Geophys. Res., 102, 7399-7410, 1997.

Sinha, H. S. S. and Raizada, S.: Some new features of ionospheric plasma depletions over the Indian zone using all sky optical imaging, Earth Planets Space, 52, 549-559, 2000.

Soden, B. J.: The diurnal cycle of convection, clouds, and water vapor in the tropical upper atmosphere, Geophys. Res. Lett., 27, 2173-2176, 2000.

Thomas, R. M., Cervera, M. A., Eftaxiadis, K., Manurung, S. L., Saroso, S., Effendy, Ramli, A. G., Salwa Hassan, W., Rahman, H., Dalimin, M. N., Groves, K. M., and Wang, Y.: A Regional GPS Receiver Network for Monitoring Equatorial Scintillation and Total Electron Content, Radio Sci., 36, 1545-1557, 2001.

Thomas, R. M., Cervera, M. A., Ghaffar Ramli, A., Effendy, Totarong, P., Groves, K. M., and Wilkinson, P. J.: Seasonal Modulation of GPS performance due to equatorial scintillation, Geophys. Res. Lett., 31, L18 806, 2004.

Tian, B., Soden, B. J., and Wu, X.: Diurnal cycle of convection, clouds, and water vapour in the tropical upper troposphere: Satellites versus a general circulation model, J. Geophys. Res., 109, D10 101, doi:10.1029/2003JD004117, 2004. 
Tsunoda, R. T.: Control of the seasonal and longitudinal occurrence of equatorial scintillations by the longitudinal gradient in integrated $E$ region Pedersen conductivity, J. Geophys. Res., 90, 447-456, 1985

Van Dierendonck, A. J., Klobuchar, J., and Hua, Q.: Ionospheric scintillation monitoring using commercial single frequency C/A code receivers, in: Proceedings of the Institute of Navigation GPS-93, pp. 1333-1343, Inst. of Navig., Alexandria, Va., USA, 1993.
Van Dierendonck, A. J., Hua, Q., Fenton, P., and Klobuchar, J.: Commercial ionospheric scintillation monitoring receiver development and test results, in: Proceedings of the Institute of Navigation GPS-96, pp. 573-582, Inst. of Navig., Alexandria, Va., USA, 1996.

Whitehead, J. D.: Ionization disturbances caused by gravity waves in the presence of an electrostatic field and background wind, J. Geophys. Res., 76, 238-, 1971.

Yang, G.-Y. and Slingo, J.: The diurnal cycle in the tropics, Monthly Weather Review, 129, 784-801, 2001. 